\title{
Niveles de diagnóstico en los procesos de inserción y orientación profesional
}

\section{Trinidad Donoso, María Pilar Figuera}

Departamento de Métodos de Investigación y Diagnóstico en Educación, Universidad de Barcelona

\section{España}

pfiguera@ub.edu 


\section{Resumen}

La orientación profesional se ha revelado como una herramienta clave para ayudar en los procesos de inserción laboral. El objetivo de este artículo es ofrecer un modelo innovador de intervención que contemple el vértice: mercado de trabajo, tejido social y contexto personal, dentro del cual se pueden entender y analizar, de una manera global, las actuaciones dirigidas a favorecer el acceso al mercado de trabajo de colectivos y personas.

El estudio ofrece indicadores que pueden ser utilizados para aplicar de una manera concreta a los procesos de intervención para la inserción.

Palabras Clave: Inserción socio-laboral, orientación profesional, diagnóstico de los procesos de inserción, intervención psicopedagógica 


\section{Introducción}

Los servicios vinculados al empleo, sean públicos o privados, se encuentran con la necesidad de tener referentes operativos para ayudar y/o promover la inserción laboral de personas o colectivos. Este artículo pretende ofrecer un marco amplio a partir del cual establecer indicadores para el diagnóstico de la empleabilidad tomando como base los tres grandes ejes de la inserción: el mercado laboral, el tejido social y la persona.

Diagnóstico para la inserción es un término comprensible y asequible socialmente, pero que puede inducir a engaño si se limita precisamente a esta acepción social de acceso al mundo del trabajo. Nosotras intentaremos descubrir todas las vertientes en que este campo se ramifica, abrir las perspectivas que le son propias y concretar en lo máximo de lo posible sus aplicaciones practicas. El diagnóstico para la inserción se inscribe dentro de la disciplina del diagnóstico en orientación profesional que se ocupa de la comprensión, análisis e intervención, de y sobre personas y grupos, enfrentadas a situaciones personales y profesionales que tienen que ver con el desarrollo de la carrera profesional.

\section{¿En qué consiste la inserción socio-laboral?}

Durante las últimas décadas se ha observado un auge importante en el estudio de los procesos de inserción laboral y social de jóvenes y adultos, desde el momento en que la orientación profesional (y de manera más amplia, la intervención psicopedagógica) ha sido reconocida como una estrategia clave en el marco de las actuaciones dirigidas a favorecer la inserción/inclusión o, lo que es lo mismo, evitar la exclusión laboral y social.

La bibliografía sobre el tema es tan exhaustiva como la amplia terminología que ha suscitado y que de manera breve clarificamos a continuación:

- El concepto de inserción laboral ha tenido gran aceptación en el marco del empleo. En numerosas ocasiones identificamos la inserción laboral con el logro de una ocupación y es habitual ver una relación directa entre salida del desempleo e inserción. Sin embargo, hoy todos los autores están de acuerdo en señalar el error de esta equivalencia. Como señala Vicens (1999), para que podamos hablar de inserción hace falta una situación relativamente estable de permanencia en la ocupación por cuenta propia o 
ajena, de manera que se consiga una autonomía económica, con una probabilidad alta de poder mantenerla. Las medidas actuales para el empleo no buscan sólo la ocupación sino la calidad de la inserción como un medio fundamental para incrementar la competitividad de los estados (ver la Estrategia Europea para el Empleo, (DO L197 de 5.8.2003).

- Establecer la calidad de vida como medida multidimensional de la inserción tiene diversas implicaciones en la acción y la evaluación de la misma. Los centros y/o los agentes de inserción han de implicarse en una acción más compleja que la simplemente de formación u otros indicadores unidimensionales para acciones que lleven a cabo. Hay que animarlos a pensar, o a repensar, en otras formas de participación social que las ligadas exclusivamente al empleo. De ese modo, los indicadores de evaluación se amplían a otros elementos que no sean puramente el haber conseguido o no trabajo, y se abren perspectivas para la acción social.

- El concepto de transición ha tenido una mayor difusión en el contexto de las políticas de formación, que se explica en parte por el gran distanciamiento que existe entre la cultura escolar y la cultura sociolaboral. Pero esta situación le da, a la vez, una dimensión de progreso, desde la adolescencia al mundo adulto y a la vida activa. Desde esta perspectiva el concepto de transición al trabajo se define actualmente como un proceso amplio, en el cual coinciden procesos de cualificación profesional y de madurez personal, condicionados por la estructura formativa y laboral (Casals, 1997, Figuera, 1996). Un proceso que se inicia en el trayecto formativo, en el que el estudiante da forma a su currículum, gesta actitudes y comportamientos, elabora estrategias y toma decisiones; y que finaliza, más allá del acceso al primer empleo, con la consolidación de la trayectoria laboral. Términos como transición a la vida activa, transición escuelatrabajo, forman parte ya del acervo lingüístico común en nuestro entorno.

- La integración sociolaboral, es uno de los conceptos que ha surgido fruto de la especificidad de contextos de intervención con poblaciones con necesidades educativas especiales o con especiales dificultades de inserción, como los emigrantes, o también las personas con disminución física o mental donde la integración laboral ha sido un elemento clave para la integración social (Jurado, 1990)

- El continuo inserción-exclusión. El concepto de inserción suele también definirse en relación con el de exclusión, considerados como excluyentes y a la vez como definitorios de un único proceso. El concepto de exclusión, (como sus homónimos de marginación o vulnerabilidad) indica el polo más negativo del continuo exclusión - inser- 
ción, y es un concepto que se ha identificado con el proceso de aparcamiento de los ámbitos sociales propios de la comunidad en la que se vive, o como una pérdida de autonomía para conseguir los recursos necesarios para vivir, integrarse y participar en la sociedad de la que forma parte (Ballester y Figuera, 2000).

- El sistema de transición. Tanto la inserción como la exclusión están relacionadas con la estructura de la sociedad concreta en la que se desarrollan sus dinámicas. La forma en que la sociedad está organizada y estructurada, el desarrollo de los sistemas formales de formación, el papel de las políticas de ocupación, los sistemas de ayuda a las personas que están en este proceso, tienen una influencia significativa en las trayectorias de transición y en el tipo y distribución de las posibilidades o de las dificultades sociales. El concepto de "sistema de transición" (o inserción según el término), incluye el conjunto de acciones públicas y dispositivos acerca de la transición (Rose, 1987; Casal, 1997).

- El itinerario. Uno de los conceptos más arraigados en el tema de la inserción es el de itinerario (Rodríguez Moreno, 1999), que cabe situar en el marco de la biografía de la persona y que define el trayecto o proceso que realiza una persona mientras planifica, organiza y construye el diseño de los diversos roles que asume en su vida adulta. Conlleva gestionar las propias posibilidades combinándolo con los medios que ofrece el ambiente.

\section{¿Qué es diagnóstico para la inserción?}

El diagnóstico para la inserción tiene como objetivo ayudar y asistir a las personas para poder hacer frente a las exigencias sociales y profesionales que plantea el acceso al mercado de trabajo, desde los comienzos en la preparación para incorporarse a la vida activa, pasando por la exploración del mundo profesional, la inserción y adaptación al primer empleo, los reajustes que se ocasionan en la vida profesional -despido, abandono, nueva búsqueda de trabajo-, y los reajustes en los roles vitales-profesionales motivados bien por los propios roles, bien por situaciones laborales.

Este proceso conlleva analizar variables, interpretarlas, combinarlas en un esquema lógico y establecer planes de acción. Una gran mayoría de estas variables necesitan del substrato psicológico que es lo que nos permite comprender su estructura, como si de un ADN se 
tratara, pero el proceso que se lleva a cabo es eminente y fundamentalmente un proceso educativo. (Donoso, 2001).

\section{Características del diagnóstico para la inserción}

En el marco de la intervención dirigida a la inserción de jóvenes y adultos se hace necesario apuntar una serie de características que: a) diferenciarán este tipo de diagnóstico de otros que puedan realizarse dentro del ámbito escolar o con poblaciones infantiles; b) marcarán el proceso y c) guiarán las actuaciones del profesional o profesionales implicados (Donoso, 1998)

- Las decisiones en la vida adulta adquieren un cariz completamente diferente a las que se hayan podido tomar anteriormente. El término que mejor representa la toma de decisiones en la vida adulta es el de "vinculante", muy utilizado en terminología legal y aquí entendido como los compromisos que se adquieren y las exigencias que plantea para la persona cada una de las decisiones tomadas. La elección de un camino equivocado o erróneo en la vida adulta que sea necesario reajustar conlleva un gasto adicional de energía con un "coste" importante para el sujeto. La persona tiene siempre presente este punto aunque sea a un nivel inconsciente, lo que convierte la toma de decisiones en un tema mucho más acuciante, ansiógeno y significativo que en cualquier otro momento de la vida. Ahora bien, esta característica que el profesional debe tener presente, ha de combinarse con otras dos características que también comentaremos: la incorporación de los errores en el proceso diagnóstico y el desligarse del estereotipo de la edad.

- El estereotipo de la edad o edadismo (Padilla, 2001) es la tendencia a juzgar las conductas de un ser humano y valorarlas, como apropiadas o no, en función de las normas sociales para una edad determinada. Las trayectorias vitales y profesionales se están ampliando y cambiando de una manera vertiginosa, la misma rapidez que se observa en la evolución de las profesiones o en los cambios sociales. Por ello, los indicadores del diagnóstico infantil, en el cual es necesario tener unos referentes evolutivos y conocer las características del grupo de edad como guías para las interpretaciones, no se justifican en el diagnóstico con adultos. 
- El aprendizaje por ensayo y error está presente en el proceso de aprendizaje en general y deberíamos aplicarlo por igual al desarrollo de la carrera; entre otras cosas es de notar que las personas conceden a sus fracasos una gran importancia, con una carga emocional alta. Introducir el valor del error en el diagnóstico en orientación profesional requiere un replanteamiento de los procesos lineales utilizados en dicho diagnóstico.

- En los procesos formativos el diagnóstico debe intentar combinarse en un programa conjunto en el cual algunos elementos de este último queden vinculados al proceso formativo. Imaginemos un curso de formación en un colectivo de riesgo: parados de larga duración, exreclusos, etc; recabar datos para el diagnóstico puede hacerse a través de la inclusión de actividades dentro de las áreas curriculares, como expresar mediante escritos anotaciones sobre su vida, sus personajes de referencia, sus puntos fuertes y débiles...; elementos que después se recogerán como indicadores diagnósticos. En la misma forma las interpretaciones diagnósticas deberían, siempre que fuera posible, introducirse en el proceso formativo.

- La orientación para la inserción, y por esto mismo el diagnóstico, debe realizarse desde el contexto concreto donde viven las personas. La presentación de vías alternativas de formación ha de haber estudiado con antelación el contexto ambiental en que éstas se mueven ya que carece de sentido realizar una intervención en abstracto. Ha de prestarse especial interés a las entidades y organizaciones enclavadas en el entorno, a las conexiones con la comunidad y a otros agentes orientadores, insertores o formadores, que actúen en ese contexto.

- ¿Cuáles son las variables relevantes a analizar en cada caso concreto?. El marco teórico de actuación indica las variables relevantes del diagnóstico a ser tenidas en cuenta. En virtud de la amplitud de este marco teórico se analizarán las áreas que pueden incidir en la inserción.

En otra publicación anterior (véase Donoso y Figuera, 2001), nos hemos posicionado en lo que se considera uno de los aportes teóricos más influyentes: la perspectiva sociocognitiva, un enfoque comprensivo desde el cual explicar los procesos de diagnóstico e intervención y que tiene su origen en el enfoque cognitivo-conductual. Dentro del enfoque cognitivo-conductual se incluye hoy toda una línea de planteamientos que inicialmente pueden proceder de dos enfoques diferenciales, el neoconductismo y el cognitivismo y que actualmente se consideran como dos líneas que se han complementado. Ambas corrientes han evoluciona- 
do hasta emerger los planteamientos cognitivistas del desarrollo de la carrera desde la perspectiva constructivista que hoy domina el campo de la explicación e intervención sobre la conducta humana. La teoría socio-cognitiva de la carrera (SCCT), formulada por Lent, Brown y Hackett (1994), aplica al campo de la orientación profesional los principios fundamentales de la Teoría socio-cognitiva de A. Bandura (1990), recoge también las aportaciones de las teorías cognitivas de la motivación del trabajo. Un trabajo posterior de los autores les ha permitido aplicar los conceptos a los procesos de transición al trabajo (Lent, Hackett y Brown, 1999).

El enfoque conceptualiza la transición al trabajo como un proceso que se desarrolla gradualmente a través del itinerario de formación, de manera que la entrada en el mundo laboral viene precedida de un largo período preparatorio y seguido por un período extensivo de preparación y ajuste al trabajo y a otros roles vitales.

La realización del proyecto profesional es una empresa cognitiva, condicionada por un interjuego de factores personales y sociales. La persona es un agente activo que crea y forma su ambiente; la teoría sostiene que las personas perciben, sopesan e incorporan información selectivamente acerca de ellos mismos y del ambiente cuando consideran varios patrones de carrera, formulan metas personales y profesionales que ayudan a impulsar su conducta de carrera en base a sus creencias acerca de sus capacidades y otros auto-atributos, de los recursos aportados por su ambiente y de la probabilidad de resultados de los diferentes cursos de la acción (Lent, Brown y Hackett, 2000).

- Las técnicas e instrumentos utilizados deben adecuarse al colectivo concreto con el que estemos trabajando. Debemos establecer que los mínimos criterios para la elección de instrumentos pasa por (Padilla, 2002):

- Elegir los que se corresponden con el nivel de procesamiento de la información que tienen los sujetos.

- Escoger aquellos que sean capaces de captar la diversidad de situaciones de inserción que puedan darse e incluso la diversidad de personas y grupos.

- Atender al nivel de capacidad comunicativa que posea el sujeto para inclinarse por uno u otro en función de cómo ponga en juego esa capacidad.

- Tener presente los sesgos y limitaciones propias del instrumento en sí, y 
- Escoger aquellos con los que se haya adquirido una cierta familiaridad (Buendía, 1994).

- La implicación del adulto es básica para conseguir integrarlo en las guías de intervención que se lleven a cabo y en el cambio imprescindible que toda intervención lleva emparejada. Es necesario que todo el proceso sea motivador, consiga despertar el interés de los implicados y pueda mantener en ellos un nivel adecuado de energía hasta el final del proceso.

\section{Niveles de diagnóstico en los procesos de intervención para la inserción}

El diagnóstico es una estrategia fundamental que acompaña y forma una parte integral de la acción orientadora en las diferentes etapas de la intervención (Sobrado, 2005). Pensar en el diagnóstico como una estrategia integrada en el proceso de orientación, obliga a tomar como punto de referencia el propio papel del profesional de la orientación en el marco de la etapa en la que se pretende intervenir: la inserción en el mercado de trabajo en el caso que nos ocupa.

Desde la perspectiva de un modelo global de intervención de los procesos de inserción, se deben considerar de manera articulada tres vértices que representan los elementos implícitos en la resolución de los procesos de inserción: el mercado de trabajo, la comunidad y la persona. ¿Qué implica este hecho?; ¿por qué el técnico de orientación, que vemos como un profesional que trabaja con personas, bien en procesos de asesoramiento individualizado o en grupo, debe valorar algo más que la dimensión biográfica o personal?; cuando hablamos de inserción ¿estamos hablando de un proceso complejo en el que, más que nunca, no podemos tener sólo en cuenta la persona y su atención desde el servicio en que nos encontremos?

No se pueden atender a las necesidades individuales de la persona si no se sabe cómo funciona la dinámica del mercado laboral, o hacia donde va la ocupación; cuáles son los puntos de atención de las políticas de ocupación o qué políticas fiscales o laborales favorecen o dificultan la información. Estas son algunas preguntas a plantearse, pero hay otras más que tienen que ver con el contexto próximo en el que se trabaja, y, así, deberemos conocer qué piensan y cómo actúan las empresas, específicamente las relacionadas con el mercado de tra- 
bajo específico de cada colectivo; y, al mismo tiempo, qué ejes de desarrollo tienen los diferentes marcos de actuación.

Como se representa en la figura 1, nos encontramos frente a dos niveles diferentes de intervención: el macro, o contexto donde las personas realizan el transito a la ocupación y el micro, referido a la persona en si. La persona constituye el vértice de la intervención, mientras que el mercado laboral y el tejido social serán los dos referentes-recurso para poder facilitar el logro de los objetivos concretos en referencia a la ocupación.

En cada uno de ellos debemos integrar el diagnóstico y las otras estrategias orientado ras.

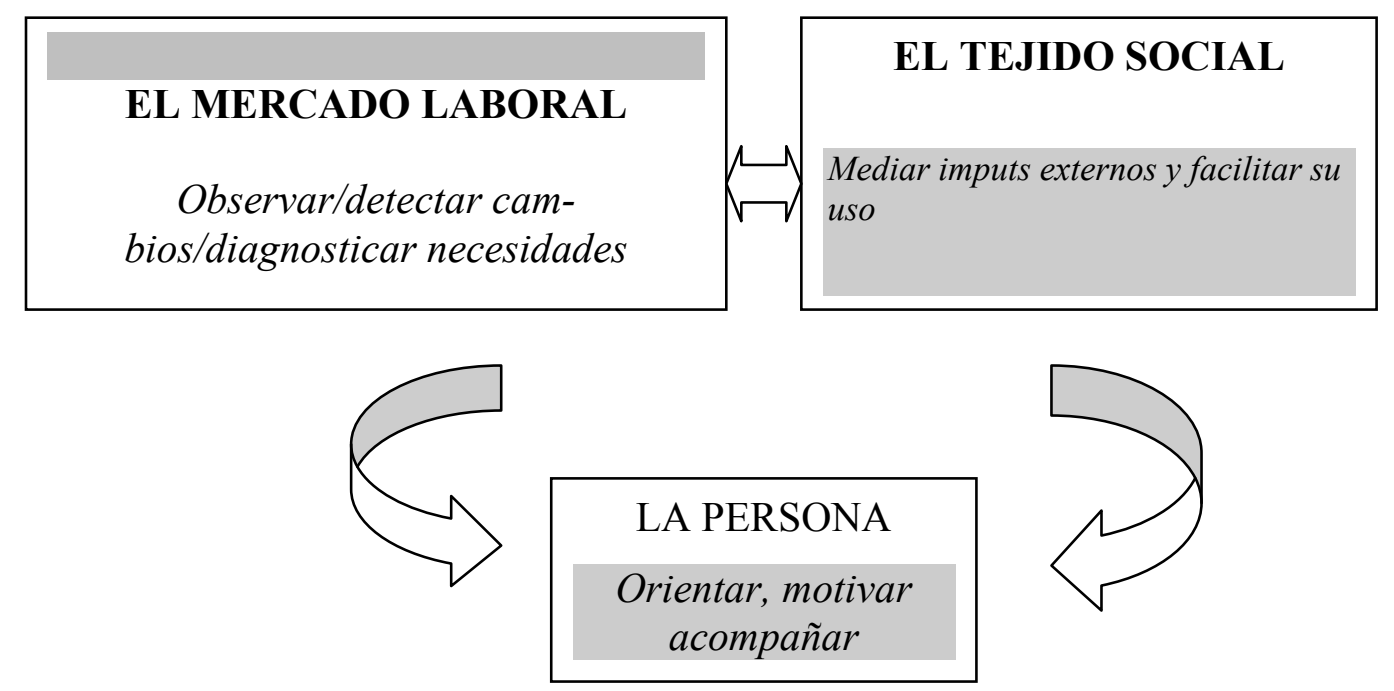

Figura 1. Referentes del proceso diagnóstico

\section{El diagnóstico macro: Mercado laboral y tejido social}

Así, los vértices superiores recogen aquellos elementos externos a la persona pero que condicionan el éxito de la intervención. A continuación detallamos algunas de las cuestiones clave: 


\section{El mercado laboral}

El profesional que actúa de mediador de los procesos de inserción de los diferentes colectivos en transición a la ocupación ha de tener una información referencial sobre la evolución del mercado de trabajo y ha de conocer las características y los indicadores de cambio específico relacionados con las posibilidades laborales de los usuarios. El "diagnóstico" de estas variables permite al profesional valorar las posibilidades de inserción de las personas en general y, a la vez, detectar problemáticas específicas de determinados colectivos.

\section{ELEMENTOS DE DIAGNÓSTICO del MERCADO DE TRABAJO}

\section{DINÁMICA DEL MERCADO DE TRABAJO}

Dinámica de mercado de trabajo

Nivel de concertación
Características y sectores de actividad

Crecimiento y desaceleración: fluctuaciones del mercado

Existencia y funcionamiento de los Pactos territoriales para la ocupación

Dinámicas de desarrollo (regional, local, ...)

\section{RELACIÓN ENTRE LA OFERTA Y LA DEMANDA DE TRABAJO}

Características de la población en paro (oferta de trabajo)

\section{Demanda}

Cuadro1: Dimensiones de diagnóstico del mercado de trabajo. Fuente: Diagnóstico de la transición escuela-trabajo. Documento policopiado. (Figuera, Freixa, Garcia, 2000). Universidad de Barcelona.
Datos desagregados de ocupación en función de variables como la edad, el sexo, la procedencia geográfica, el nivel educativo

Adecuación de las competencias profesionales a los requerimientos del mercado

Disponibilidad: movilidad, flexibilidad horaria, salarial, contractual....

Inactividad/paro

\section{Requisitos}

Tipologías de empresas: tamaño, NTT....

Condiciones de trabajo

Co-responsabilidad en el desarrollo del territorio: concertación, planificación... 
Tanto en el marco formativo como en el laboral, deben de tenerse en cuenta las peculiaridades del entorno sociocultural, tecnológico y económico propio. El cuadro anterior presenta algunos elementos a tener en cuenta, cuando se parte de la definición de mercado de trabajo como el espacio de intercambio entre oferta y demanda.

\section{El tejido social}

El tejido social agrupa el conjunto de recursos comunitarios, organizativos y de sorporte social (red de transición) pensados o diseñados para amortiguar el impacto de las situaciones críticas y de factores de riesgo relacionados con las trayectorias de exclusión. La comunidad dispone hoy de una red social dirigida a facilitar la transición de las poblaciones en paro al trabajo. Ahora bien, sabemos que la vulnerabilidad de los diferentes colectivos a las situaciones críticas y a los factores de riesgo de exclusión está relacionada con la disponibilidad, accesibilidad y adecuación de los recursos a sus necesidades y demandas (García y Figuera, 2000). Por ello, la finalidad fundamental del proceso de intervención es mediar entre los inputs y recursos que la comunidad establece y las personas, a partir de identificar los recursos materiales y humanos de la zona o sector para su mejor aprovechamiento y facilitar su uso, a la vez que canalizar la intervención de los servicios y equipos de apoyo a la transición, sobre todo en situaciones multiproblemáticas.

Desde el marco social el diagnóstico tiene un papel fundamental. Debe ir dirigido a recabar información sobre las prioridades de las políticas institucionales (locales, autonómicas,....), tanto desde la perspectiva de las acciones como de los propios destinatarios de la intervención.

Del diagnóstico de la red social para la transición al empleo debemos obtener información para articular el diseño de la intervención:

- ¿La red de recursos para la transición a la ocupación contempla las necesidades diferenciales de los diferentes colectivos en demanda de ocupación?

- ¿En qué medida se actúa coordinadamente en los contextos más próximos a los usuarios y se prioriza la prevención?

- ¿De qué manera estas acciones pueden fortalecer las diversas redes de trabajo, intra o extramunicipal, iniciadas en el municipio? 
- ¿Qué elementos pueden contribuir a dar una mayor operatividad a la red social de ayuda a la ocupación? ¿en qué medida la situación permite formalizar instrumentos de trabajo para favorecer la coordinación?

\section{El diagnóstico micro: el vértice personal}

Si hemos ofrecido indicaciones para el diagnóstico del mercado de trabajo y del tejido social, debemos ahora situarnos en el nivel personal, último apartado del bloque conjunto desde el que cabe realizar el diagnóstico para la inserción. Puesto que son muchas las variables que pueden analizarse, nos preguntamos cuáles pueden ser entendidas como fundamentales y sobre todo cuál es el objetivo que tienen en el plano de la inserción.

\section{Identidad laboral}

¿ Cómo se conceptualiza la persona al verse en su rol de trabajador/a?. Creemos que aquí se incluyen tanto las preferencias que demuestra hacia las distintas ocupaciones y profesiones como la percepción que realiza de sus competencias (Figuera, 1996).

¿Cuál es el objetivo del diagnóstico de los intereses y cuáles son los condicionantes a considerar en el diagnóstico?

Conceptualizada en una primera época como intereses ocupacionales, el objetivo clásico de rastrear las preferencias que demuestra una persona por ciertas ocupaciones y ajustar sus elecciones a esas preferencias ha evolucionado hacia otros tipos de objetivos: a) utilizar el diagnóstico como estímulo que permita discutir e incrementar la autoconciencia del propio sujeto; b) como marco comprehensivo que sirva de indagación e investigación sobre las diferentes ocupaciones; y c) como consideración de alternativas profesionales con un efecto retroactivo sobre el propio sujeto.

El rastreo de los intereses de una persona o grupo ha de considerar diversos elementos que son generalizables a cualquier situación de diagnóstico en la actualidad: la evaluación no es un medio de clasificación rígida en ningún tipo de categoría; no debe realizarse para dirigir a la persona según crea el profesional que esté realizando el diagnóstico. Los resultados deben ser discutidos y analizados con el interesado, más específicamente en el caso de adolescentes 
y adultos; la última palabra sobre los análisis realizados en un área determinada y el significado que se le otorgue la tiene siempre la propia persona.

En el caso específico de los intereses habría que preguntarse también sobre otros elementos durante la evaluación: ¿el significado de las ocupaciones tiene una base realista para la persona?, es decir, ¿entiende exactamente lo que son o las percepciones que tiene sobre ellas están influidas por elementos imaginativos provenientes de sesgos sociales?, ¿qué elementos concretos pueden estar afectando la elección de determinadas profesiones u ocupaciones profesionales: el género, el imaginario social, etc.?, ¿entiende la persona que la evaluación no debe convertirse en un determinante de sus decisiones sino en un proceso de reflexión a combinar con otros elementos?. Todas estas preguntas nos llevan a considerar que la evaluación de intereses no puede convertirse en un elemento puntual de actuación sobre un grupo, como se ha realizado mayoritariamente hasta ahora, sino en un elemento procesual de ayuda a los sujetos que tiene que contar con una información completa anterior a la evaluación, un proceso de evaluación que puede y debería repetirse y una orientación posterior de comentario y análisis de los resultados.

¿Qué significado tiene la percepción de las competencias?

La autoeficacia personal siempre va dirigida a un dominio de competencia. Esta percepción sobre la propia capacidad va a ser fundamental a la hora de que el trabajador ponga en marcha una serie de competencias sociales y profesionales. Tal como han demostrado las investigaciones, la autoeficacia actúa en sentido inverso: frena la inserción cuando es negativa aunque no necesariamente la activa cuando es positiva, seguramente por el efecto modelador en relación a otras variables. Consecuentemente también va a modular la cantidad de energía que se utiliza de manera efectiva en el cumplimiento de la tarea (búsqueda de empleo, formación específica...); de alguna forma es como si su signo positivo liberase la energía que permite emplearse en acciones concretas y que de otra manera quedaría bloqueada.

\section{¿Por qué y para qué trabajar?}

Las metas que se plantea el futuro trabajador van a afectar a la intensidad y la persistencia de las conductas que se realicen. Estas metas pueden ser varias: ganar dinero, hacer amigos, tener un trabajo interesante o conseguir un mayor nivel de bienestar (Russell, 1932). Cuando están 
centradas en la tarea tienen un grado de activación distinto que cuando están centradas en otros elementos colaterales o consecuentes al tipo de tarea o trabajo en sí, pero es natural que esta variable interactúe con la infraestructura personal, como es la formación, el género o la clase social entre otras.

¿Existe una personalidad que tenga que ver con la competencia en y para el trabajo?

El tipo de personalidad está muy ligada al tipo de tarea que deba realizarse y a las condiciones en que deba realizarse. Es por eso que hoy día interesa evaluar las características propias de la personalidad ligadas a estos factores. No existe un "perfil" mejor que otro, sino que cada característica va unida al tipo de trabajo y a los compañeros con los que se realiza; de cara a la intervención es mas fácil trabajar desde lo que uno es que desde lo que puede o debe cambiar. Al mismo tiempo estas características interactúan con las habilidades sociales que se ponen en juego dependiendo de las situaciones determinadas que se den en el lugar de trabajo. Se acepta de manera bastante general que existen cinco grandes factores que explicarían los rasgos de personalidad; de entre ellos las empresas están interesadas por la honradez, la escrupulosidad, la tolerancia al estrés y control del enfado y la preferencia por las relaciones con los otros (véase la revisión completa de Rivas (1995) al respecto y Liénard, 2001). Sin embargo, las características de personalidad ligadas a los procesos de inserción están dando un vuelco debido a que parece más interesante analizar e intervenir sobre estilos de procesamiento de la información que sobre características de personalidad como tal (Hoffman, 2002). Quizás no sea tan interesante evaluar la personalidad, sino establecer cómo la persona procesa, enfoca, observa, atiende a las normas. Estos serían los elementos que interactuarían con la competencia del futuro trabajador. A este respecto no hay que olvidar las aportaciones que se están realizando desde el concepto de inteligencia emocional, ligando las reacciones más ligadas a la personalidad al constructo inteligencia (Mayer y Salovey, 1993,1995; Mestre Navas, et al., 2005).

Al mismo tiempo la variable ansiedad sigue teniendo especial relevancia por cuanto modula las características de la personalidad. 
¿Y qué hay del nivel de ocupabilidad?

Aunque de manera latente una determinada acreditación o titulación lleva emparejada la presunción de un determinado nivel de inteligencia o el nivel de ocupabilidad de la persona. Esta situación perjudica a las personas con niveles bajos de cualificación; un error que intenta subsanarse con la entrada en el mercado laboral de los modelos de competencias profesionales, en los cuales la trayectoria profesional, y por tanto, experiencial del sujeto, pasan a considerarse de manera relevante (Echeverría, 2005). De todas formas no hay que olvidar que la formación recibida cuando va acompañada de credenciales educativas ofrece seguridad y confianza para enfrentarse a los procesos de búsqueda de empleo.

Altos o bajos niveles de formación no son por sí mismos indicativos de mayor o menor facilidad para encontrar empleo, mientras que la capacidad de aprendizaje del futuro trabajador permite constatar si superará las pruebas de selección a las que se vea sometido, el tiempo de prueba en el futuro puesto de trabajo o el éxito en los cursos de formación ocupacional tanto en los aspectos teóricos como prácticos.

En los colectivos con semi o baja cualificación el diagnóstico debería centrarse en las posibilidades de resolución de problemas, aislando los apartados que pertenecen exclusivamente al currículum escolar, ya que los niveles tan bajos que pueden presentar estas personas enmascara sus verdaderas posibilidades intelectuales.

\section{Algunas consideraciones sobre el proceso}

No existe un modelo claro para el desarrollo del proceso diagnóstico en inserción profesional, al contrario de lo que ocurre en los variados modelos de procesos tanto para el diagnóstico educativo como para el psicopedagógico o incluso para los de intervención en orientación profesional. Con esto no queremos decir que no se hayan delineado guías en este sentido pero no pueden aplicarse específicamente al tema de la inserción sino más ampliamente al diagnóstico en orientación profesional.

A la luz de lo que sabemos hoy en este campo creemos que la aplicación del diagnóstico debe guiarse al menos por los siguientes principios: 
Planificación flexible y abierta

Los procesos de pensamiento involucrados en la gestión de la carrera profesional tienen cada día más relevancia. Como profesionales nos interesa conocer cuáles son los procesos de pensamiento que garantizan una gestión eficaz de la carrera. Entre las competencias relacionadas con este tema queremos destacar una: ayudar a pensar en un amplio repertorio de conceptos relacionados con las elecciones, roles, funciones, etc. que se dan en la carrera profesional y cómo estos conceptos se combinan entre sí. Nos planteamos hasta qué punto los sistemas secuenciales de diagnóstico permiten poder acercarse al análisis de estos procesos interactivos y complejos. Descubrir cuáles son los procesos de pensamiento de las personas involucradas en el diagnóstico con las técnicas actuales resulta bastante difícil, aunque ya se están ensayando procedimientos al respecto.

Activar y desarrollar el proceso de aprendizaje, paradigma de actuación.

Adquirir un trabajo, acceder a él, mantenerse, prepararse para la vida profesional, integrarse en la realidad laboral, etc. Todo ello puede depender de muchos factores cuyo único denominador común es el aprendizaje. El éxito o no de la vida social de una persona que lleva emparejado el acceso a la vida activa pasa por su capacidad de aprendizaje, una capacidad que debe ser o estimulada, o desarrollada o activada, dependiendo de la historia personal de cada uno, por medio del proceso diagnóstico.

El aprendizaje debe incorporar la resolución de problemas, con lo que además integra la parte cognitiva y volitiva de la persona. Así, no sólo es conocer o comprender, sino además actuar en un sentido determinado, impulsar a la acción, producir un cambio.

\section{El valor de la "equivocación"}

El aprendizaje por ensayo error está presente en el proceso de aprendizaje en general y deberíamos aplicarlo por igual al desarrollo de la carrera. Entre otras cosas cabría señalar que las personas conceden a sus fracasos una gran importancia con una carga emocional alta. Introducir en el diagnóstico en orientación profesional el valor del error requiere un replanteamiento de los procesos lineales utilizados en el diagnóstico. 
No importa dónde se sitúe sino dónde puede llegar

En toda la línea cognitiva de diagnóstico en educación se trabaja con la psicología vigostkiana de la zona de desarrollo próximo. Primero sabemos que las influencias ambientales pueden marcar un límite a la capacidad de pensamiento y estilo de una persona; y segundo, se trata de analizar no sólo dónde está el sujeto actualmente sino dónde puede llegar. Y así ayudar, mediante el diagnóstico, a desarrollar esta zona de desarrollo potencial. Este mismo planteamiento deberíamos aplicarlo al diagnóstico en orientación profesional. No sólo debe interesarnos con lo que el sujeto cuenta en relación con las variables medidas, sino su potencialidad para llegar a desarrollar tales variables y convertir el proceso de diagnóstico en un medio para hacerlo. Hasta ahora este procedimiento se ha aplicado con éxito al desarrollo cognitivo, sin embargo, ¿por qué no averiguar sus posibilidades en otro tipo de variables?, ¿por qué no intentar su aplicación para desarrollar el potencial en la planificación y gestión de la carrera?, y es más, ¿no puede abrirse una vía prometedora para el delicado punto de la predicción en diagnóstico?

\section{No olvidar la realidad macrocontextual de la persona adulta}

En el desarrollo de la carrera profesional comienzan a adquirir una importancia considerable los aspectos subjetivos, esto es, el sentido que los sujetos hacen de sus historias personales, y de las destrezas, aptitudes, creencias etc., que han adquirido. Pueden encontrarse multiplicidad de significados que pueden ser dados a una serie de circunstancias. Esto requiere la introducción de técnicas en el diagnóstico que permitan aprehender estos mecanismos que se están considerando esenciales independientemente la línea teórica que se siga (las historias de vida son un buen ejemplo).

\section{Un apunte final: Diagnóstico no es ya mirar y observar, sino básicamente actuar}

A lo largo de estas páginas hemos tenido ocasión de apuntar algunas reflexiones sobre el rol del diagnóstico en los procesos de inserción socio-profesional.

De una vez por todas, aunque ya se ha insistido y escrito mucho al respecto, debe acabarse con la disociación diagnóstico-intervención. No sólo porque la intervención debe acompañar al diagnóstico (este es ya un planteamiento clásico), sino porque el proceso debe ser al 
mismo tiempo intervención, considerándolo no como elemento residual, sino como elemento planificado y activo.

El diagnóstico no puede limitarse a la descripción de una realidad, por ejemplo: cómo es una persona, cómo actúa, cuáles son las características de un contexto..., sino que debe al mismo tiempo conseguir que la persona diagnosticada sea consciente de esa realidad para trabajar cómo optimizarla, superar deficiencias, desarrollar destrezas, etc. El diagnóstico y la intervención aparecen estrechamente unidos e incluso entremezclados en todo el proceso.

Hemos trabajado ya en esta línea con una escala de conducta exploratoria (Donoso, Figuera y Rodríguez Moreno, 1994; Donoso, Figuera y Torrado, 2000; Donoso y Figuera, 2001) que puede ser utilizada como un procedimiento clásico de diagnóstico: pase de la escala, corrección, valoración y devolución de resultados, pero que hemos transformado mediante una guía en un proceso donde se combinen diagnóstico e intervención, de manera que el proceso de diagnóstico de la conducta exploratoria represente para la persona una activación de las dimensiones ligadas a dicha conducta. 


\section{Referencias}

Ballester, L. y Figuera, P. (2000). Exclusión e inserción social. En P. Amorós y P. Ayerbe (Eds.) Intervención educativa en inadaptación social. Madrid: Síntesis.

Bandura, A. (1990). Perceived self-efficacy in the exercice of personal agency. Revista Española de Pedagogía, 187, 397-424.

Buendía, L. (1994). Técnicas e instrumentos de recogida de datos. En M-P. Colás y L. Buendía, Investigación Educativa.201-248. Sevilla: Alfar.

Casal, J. (1997). Modos emergentes de transición a la vida adulta en el umbral del siglo XXI: Aproximación sucesiva, precariedad y desestructuración. REIS, 75, 295-396.

Donoso, T. (1998). Diagnóstico en Educación: ¿Cuál es el pérfil que responde a las exigencias de la práctica actual? Revista d'Orientació, vol XI, 18, 23-31.

Donoso, T. (2001). Diagnóstico en orientación profesional: Controversias para el avance. . Revista Investigación Educativa, vol. 19 (2), 453-458.

Donoso, T. Y Figuera, F. (2001). El diagnóstico en la transición de los estudiantes al mercado laboral. En Alvarez, M. y Bisquerra, R. Manual de Orientación y Tutoría. Barcelona: Praxis. págs. 464/42.13-464/42.24.

Donoso, T.; Figuera, P. y Rodríguez, M.L. (1994). Un instrumento para evaluar la conducta exploratoria en el desarrollo de la carrera profesional. Revista de Investigación Educativa, 23, 490-496.

Donoso, T.; Figuera, P. y Torrado, M. (2000). Análisis y validación de una escala para medir la conducta exploratoria. Revista de Investigación Educativa, vol. 18 (1), 201-223.

Echeverría, B. (2005). Competencia de acción de los profesionales de la orientación. Madrid: ESIC.

Figuera, P. (1996). La inserción del universitario en el mercado de trabajo. Barcelona: EUB.

Garcia, M. y Figuera, P. (2000). La Garantia Social, un recurs de lluita contra la exclusió social. Temps d'Educació, 24, 241-262.

Hoffman, E. (2002). Tests psicológicos. Barcelona: Paidós.

Jurado, P. (1990) La integración socio-laboral de los sujetos con retraso mental ligero y de capacidad intelectual límite en el ámbito de la educación especial. Tesis Doctoral Inédita. Universidad Autónoma de Barcelona.

Lent, R.W. y Brown, S.D. (1996). Social cognitive approach to career development. The Career Development Quarterly , 44 (4), 310-321. 
Lent, R.W. Brown, S.D. y Hackett, G. (1994). Toward a unifying social cognitive theory of career and academic interest, choice, and performance. Journal of Vocational Behavior, 45, 79-122.

Len, R.W. Brown, S.D. y Hackett, G. (2000) Contextual supports and barriers to career choice: A social cognitive analysis, Journal of Counseling Psychology, 47 (1), 36-49

Lent, R.W. Hackett, G. y Brown, S.D. (1999). A social cognitive view of school-to-work transition. The Career Development Quarterly , 47 (4), 297-311.

Liénard, G. (ed.) (2001). L'insertion: défi pour l'analyse, enjeu pour l'action. Belgique: Mardaga.

Mestre Navas, J.M.Guil Bozal, R. y Mestre Moreno, R. (2005). Inteligencia emocional : resultados preliminares sobre su naturaleza y capacidad predictiva a partir de un estudio correlacional en mustras de estudiantes de secundaria. Revista Española de Orientación y Psicopedagogía, 16 (2), 269-281.

Meyer, J.D. y Salovey, P. (1993). The intelligence of emotional intelligence. Intelligence, 17 (2), 433-442.

Meyer, J.D. y Salovey, P. (1995).Emotional intelligence and the construction and regulations of feelings. Applied and Preventive psychology, 4(3), 197-208.

Padilla, Mª .T. (2001). Estrategias para el diagnóstico y la orientación profesional. Barcelona: Laertes.

Padilla, Mª .T. (2002). Técnicas e instrumentos para el diagnóstico y la evaluación educativa. Madrid: CCS.

Rose, J. (1987). En busca del empleo perdido. Formación, paro, empleo. Madrid: MTSS.

Rodríguez Moreno, Ma. L. (1998). La Orientación Profesional. I. Teoría. Barcelona: Ariel.

Rodríguez, Ma .L, Sandín, M.P., Buisán, C. (2000). La conducta exploratoria: concepto y aplicaciones. Revista de Educación, 321, 153-186.

Rodríguez, Mª.L. (1999). Enseñar a explorar el mundo del trabajo. Archidona, Málaga: Aljibe Ediciones.

Rivas, F. (1995). La conducta vocacional: Proceso de socialización y de desarrollo personal. En F. Rivas (ed.). Manual de asesoramiento y orientación vocacional, pp. 23-60. Madrid: Síntesis.

Russell, B. (1932) In praise of idleness. Artículo publicado en Harper's Magazine, USA, y recopilado en 1983 en el reading de V. Richards (Ed.) Why Work? Arguments for the Leisure Society, Londres: Freedom Press, págs. 25-35. 
Sobrado, L. (2005) El diagnóstico educativo en contextos sociales y profesionales. Revista de Investigación Educativa, 23 (1), 85-112.

Vicens, J. (1999). La inserción profesional de los jóvenes. En busca de una definición. Calificaciones \& Empleo, 23. 